\title{
Desmopressin for reducing perioperative blood transfusion
}

\section{Brit Long' ${ }^{1}$ \\ Michael Gottlieb²}

${ }^{1}$ Corresponding author. SAUSHEC Department of Emergency Medicine, Fort Sam Houston (San Antonio). Texas, United States of America. brit.long@yahoo.com ${ }^{2}$ Department of Emergency Medicine, Rush University Medical Center (Chicago). Illinois, United States of America. michaelgottliebmd@gmail.com

Keywords: DDAVP. Desmopressin. Perioperative. Transfusion. Platelets.

\begin{tabular}{|l|l|}
\hline NNT Color recommendation & Red (No benefit) \\
\hline Summary Heading & $\begin{array}{l}\text { Compared to placebo, desmopressin (DDAVP) does not decrease red cell transfusion, } \\
\text { all-cause mortality, or thrombotic events }\end{array}$ \\
\hline Benefits in NNT & No one was helped (no transfusion or death was prevented) \\
\hline Benefits in Percentages & No one was helped (no transfusion or death was prevented) \\
\hline Harms in NNT (NNH) & No one was harmed (thrombotic event) \\
\hline Harms in Percentages & No one was harmed (thrombotic event) \\
\hline Efficacy Endpoints & Transfusion requirement and all-cause mortality \\
\hline Harm Endpoints & Thrombotic events \\
\hline Who was in the studies & $\begin{array}{l}\text { Patients with normal platelets: } 25 \text { trials (1806 patients) of DDAVP versus placebo } \\
\text { evaluating red cell transfusion, 22 trials (1631 patients) evaluating mortality, and 29 } \\
\text { trials (1984 patients) evaluating thrombotic events } \\
\text { Patients with abnormal platelets: } 5 \text { trials (258 patients) of DDAVP versus placebo } \\
\text { evaluating red cell transfusion, } 7 \text { trials (422 patients) evaluating mortality, and 7 trials } \\
\text { (422 patients) evaluating thrombotic events }\end{array}$ \\
\hline
\end{tabular}

\section{Narrative}

Desmopressin (DDAVP) is a synthetic analog of vasopressin that stimulates the release of von Willebrand factor and promotes platelet adhesion and aggregation and is therefore typically used for the treatment of von Willebrand disease and hemophilia ${ }^{1-3}$. Some professional societies, however, recommend perioperative DDAVP for the prevention of blood loss and blood product transfusion in patients with bleeding and platelet dysfunction ${ }^{4-6}$. 
The Cochrane Review discussed here included randomized controlled trials (RCTs) evaluating adult and pediatric patients with or without platelet dysfunction who received subcutaneous or intravenous perioperative DDAVP ${ }^{7}$. Platelet dysfunction was defined as prolonged bleeding time, abnormal Platelet Function Analyzer closure times, or taking antiplatelet medications. Studies including patients with known hemophilia or von Willebrand disease were excluded. Primary outcomes included the number of patients receiving red blood cell transfusion during the procedure, or within 30 days, volume transfused, and blood loss in milliliters. Secondary outcomes included all-cause mortality within 30 days, thrombotic events, reoperations due to bleeding, bleeding events during or within 30 days, hypotension within 30 days, and quality of life.

The authors identified 65 RCTs $(n=3874)$ that met inclusion criteria. For this summary, we focused on studies evaluating DDAVP versus placebo or no treatment in patients with and without platelet dysfunction. Included studies examined DDAVP in one or two doses of 0.2-0.4 micrograms/kilogram, 20 micrograms once, or 15-45 micrograms based on weight. Timing of administration varied, including preoperative, operative, or postoperative. Settings included adult and pediatric cardiac surgery, orthopedic surgery, vascular surgery, plastic surgery, hepatic surgery, kidney biopsy, and maxillofacial surgery.

Overall, there were no significant differences in efficacy endpoints such as transfusion requirements, all-cause mortality, or thrombotic events (quality of evidence: Low). These results remained the same when the analysis was repeated in the subgroup of patients with platelet dysfunction.

In addition to thrombotic events, the Cochrane review reports on the authors' term 'clinically important hypotension,' which was more common in patients receiving DDAVP (RR: 2.9; 95\% Cl: 1.3 to 6.3; ARD: 5.9\%, NNH: 17). Unfortunately, the Cochrane review did not define clinically important hypotension, and definitions were variable and often arbitrary in the original trials. Therefore, we did not list this endpoint in the summary table.

\section{Caveats}

There was variable quality of evidence for the included studies, preventing solid conclusions. Evidence quality was moderate for blood transfusion requirement in patients with normal platelet function but low to very low for all other outcomes and populations. As a result, the review authors could not pool the data for the volume of blood transfused and blood loss. Additionally, mortality and thrombotic events were rare, and studies were underpowered to assess these outcomes. The patient populations were heterogeneous concerning age, comorbidities, and DDAVP dosing. The most commonly studied groups were adult cardiac surgery and orthopedic surgery, accounting for 51 trials, with an under-representation of other surgeries. The majority of trials were conducted over two decades ago and may not reflect current settings. Less than one-third of trials included patients receiving DDAVP preoperatively, and there were no trials evaluating patients with thrombocytopenia or coagulopathy.

Finally, most of the included trials did not provide sufficient information to assess the risk of bias.

When compared to placebo, DDAVP is associated with no clinical difference in patients with normal or abnormal platelet function in red blood cell transfusion, all-cause mortality, or thrombotic complications. Based on this evidence, we have assigned a color recommendation of Red (No benefit). Further data, however, are needed to better evaluate the role of DDAVP in current settings, those with coagulopathies or on antiplatelet medications, and administration based on platelet function or viscoelastic tests.

\section{Acknowledgements}

The authors obtained approval from Dr. Shahriar Zehtabchi for this submission, who also reviewed a draft of this manuscript before submission.

\section{Author contributions}

Gottlieb M and Long B conceived the idea for this manuscript and contributed substantially to the review's writing and editing. 


\section{Competing interests}

This review does not reflect the views or opinions of the U.S. government, Department of Defense, U.S. Army, U.S. Air Force, Brooke Army Medical Center, or SAUSHEC EM Residency Program. No financial, legal, or political competing interests with third parties (government, commercial, private foundation, etc.) were disclosed for any aspect of the submitted work (including but not limited to grants, data monitoring board, study design, manuscript preparation, statistical analysis, etc.).

\section{References}

1. EEstcourt LJ, Birchall J, Allard S, Bassey SJ, Hersey P, Kerr JP, et al. Guidelines for the use of platelet transfusions. Br J Haematol. 2017;176(3):365-94. https://doi.org/10.1111/bjh.14423

2. Keeling D, Tait C, Makris M. Guideline on the selection and use of therapeutic products to treat haemophilia and other hereditary bleeding disorders. A United Kingdom Haemophilia Center Doctors' Organisation (UKHCDO) guideline approved by the British Committee for Standards in Haematology. Haemophilia. 2008;14(4):671-84. https://doi.org/10.1111/j.13652516.2008.01695.x
3. Laffan MA, Lester W, O'Donnell JS, Will A, Tait RC, Goodeve A, et al. The diagnosis and management of von Willebrand disease: a United Kingdom Haemophilia Centre Doctors Organization guideline approved by the British Committee for Standards in Haematology. Br J Haematol. 2014;167(4):453-65. https://doi. org/10.1111/bjh.13064

4. Rossaint R, Bouillon B, Cerny V, Coats TJ, Duranteau J, Fernández-Mondéjar $E$, et al. The European guideline on management of major bleeding and coagulopathy following trauma: fourth edition. Crit Care. 2016;20:100. https://doi. org/10.1186/s13054-016-1265-X

5. Kozek-Langenecker SA, Afshari AB, Albaladejo P, Santullano $C A$, De Robertis E, Filipescu DC, et al. Management of severe perioperative bleeding: guidelines from the European Society of Anaesthesiology: First update 2016. Euro J Anaesthesiol. 2017;34(6):332-95. https://doi.org/10.1097/eja.0000000000000630

6. American Society of Anesthesiologists Task Force on Perioperative Blood Management. Practice guidelines for perioperative blood management: an updated report by the American Society of Anesthesiologists Task Force on Perioperative Blood Management*. Anesthesiology. 2015;122(2):241-75. https:// doi.org/10.1097/aln.0000000000000463

7. Desborough MJ, Oakland K, Brierley C, Bennett S, Doree C, Trivella M, et al. Desmopressin use for minimising perioperative blood transfusion. Cochrane Database Syst Rev. 2017;7(7):CD001884. https://doi.org/10.1002/14651858.cd001884. pub3 